\title{
MAGNETIC COLLAPSE AND THE BEHAVIOR OF TRANSITION METAL OXIDES: FeO AT HIGH PRESSURES
}

\section{R. E. COHEN, Y. FEI, R. DOWNS ${ }^{* *}$, I. I. MAZIN ${ }^{* * *}$; D. G. ISAAK ${ }^{* * * *}$}

"Carnegie Institution of Washington, 5251 Broad Branch Rd., N.W., Washington, DC 20015

"ASU, Tempe, AZ

*** George Mason University, Fairfax, VA and Naval Research Laboratory, Washington, D.C. ****Institute of Geophysics and Planetary Physics, University of California, Los Angeles, Los Angeles, CA, and Azusa Pacific University, Azusa, CA

\section{ABSTRACT}

Linearized augmented plane wave (LAPW) results are presented for $\mathrm{FeO}$ at high pressures using the Generalized Gradient Approximation (GGA) to study the high-spin low-spin transition previously predicted by LAPW with the Local Density Approximation (LDA) and Linear Muffin Tin Orbital (LMTO-ASA) methods within the GGA. We find a first-order transition at a pressure of about $105 \mathrm{GPa}$ for the cubic lattice, consistent with earlier LAPW results, but much lower than obtained with the LMTO. The results are generally consistent with recent Mössbauer experiments that show a transition at about $100 \mathrm{GPa}$. We also discuss the origin of the transition, and show that it is not due to electrostatic crystal-field effects, but is rather due to hybridization and band widening with pressure. Examination of experimental data and computations suggest that the high pressure hexagonal phase of $\mathrm{FeO}$ is likely a polytype between the B8 NiAs and anti-B8 AsNi structures. The former is predicted to be an antiferromagnetic metal, and the latter an antiferromagnetic insulator. Implications for geophysics are discussed.

\section{INTRODUCTION}

At low pressures transition metal ions such as ferrous iron $\left(\mathrm{Fe}^{2+}\right)$ are magnetic and highspin. In this state, ferrous iron shows complete solubility with $\mathrm{Mg}^{+2}$ in most minerals, in spite of

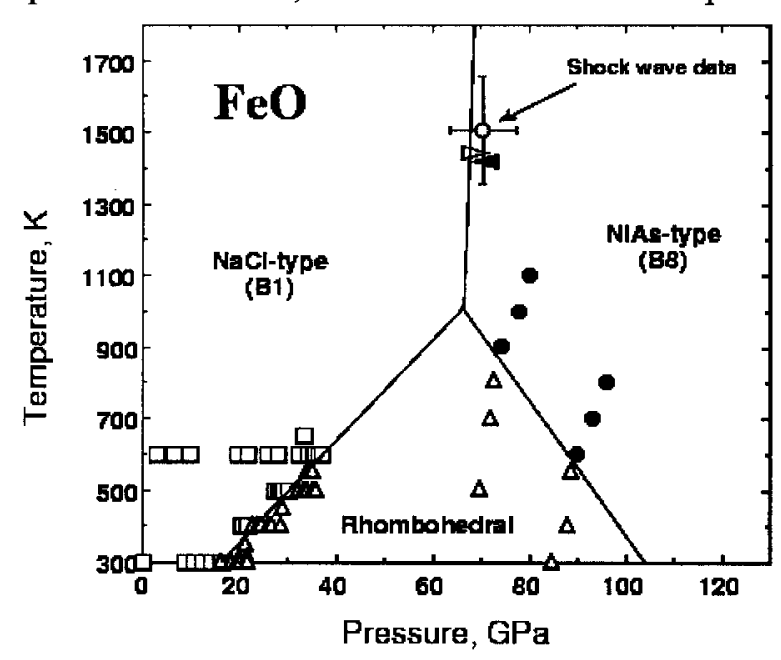

Figure 1. Simplified experimental phase diagram for FeO. After Fei (1996). the fact that $\mathrm{Mg}^{2+}$ is a simple closed shell ion, and ferrous iron is a complicated, magnetic, and non-spherical ion. In fact, $\mathrm{FeO}$ (wustite) is a Mott insulator, which is insulating due to the magnetic interactions and is given as a metal using band theory [1], and $\mathrm{MgO}$ is a simple ionic solid [2,3]. A number of transitions are expected as pressure and temperature are varied, which may occur together in groups: Mott transitions (localization transitions between band states and localized states), high spin-low spin transitions, structural phase transitions, and magnetic ordering transitions. Here we concentrate on magnetic 
high-spin low-spin transitions, and structural transitions in $\mathrm{FeO}$.

A simplified experimental phase diagram for $\mathrm{FeO}$ is shown in fig. 1. With increasing pressure there is a transition from cubic rocksalt to a strained rhombohedral structure, and at higher pressures a NiAs-type (B8) phase is observed.

Magnetic collapse was predicted at high pressures using first-principles computational methods [4,5]. Recently, experimental evidence was presented for magnetic collapse at about 100 $\mathrm{GPa}$ in $\mathrm{FeO}$ [6]. We also consider the structure of the high pressure phase by reexamining the experimental data and present firstprinciples computations for the NiAs-type phases. We also briefly discuss the geophysical and geochemical implications and give suggestions for future work.

\section{MAGNETIC COLLAPSE}

Isaak et al. [4] predicted magnetic collapse in $\mathrm{FeO}$ using the LAPW method [7] and the LDA. A transition was found at about $100 \mathrm{GPa}$. Detailed computations using the GGA (PW91) [8] and the LMTO-ASA method [9] were performed on the transition metal oxides $\mathrm{MnO}$, $\mathrm{FeO}, \mathrm{CoO}$, and $\mathrm{NiO}$ as functions of pressure [5]. The LMTO is much faster than LAPW, and at that time had GGA capabilities that were then not present in the LAPW. The LMTO results gave stable high-spin and low-spin solutions coexisting, with the high pressure phase being the low-spin phase, except for $\mathrm{NiO}$ whereas a continuous transition was found. Due to the importance of $\mathrm{FeO}$ in geophysics, we have further explored its properties using LAPW with a more recent GGA (PBE96) [10]. The LAPW computations were wellconverged; convergence parameters were 9.0 for $\mathrm{RK}_{\max }$, and 49 special k-points were used for the four-atom antiferromagnetic (AFM) phase [4], and 28 special k-points were use for the 2-

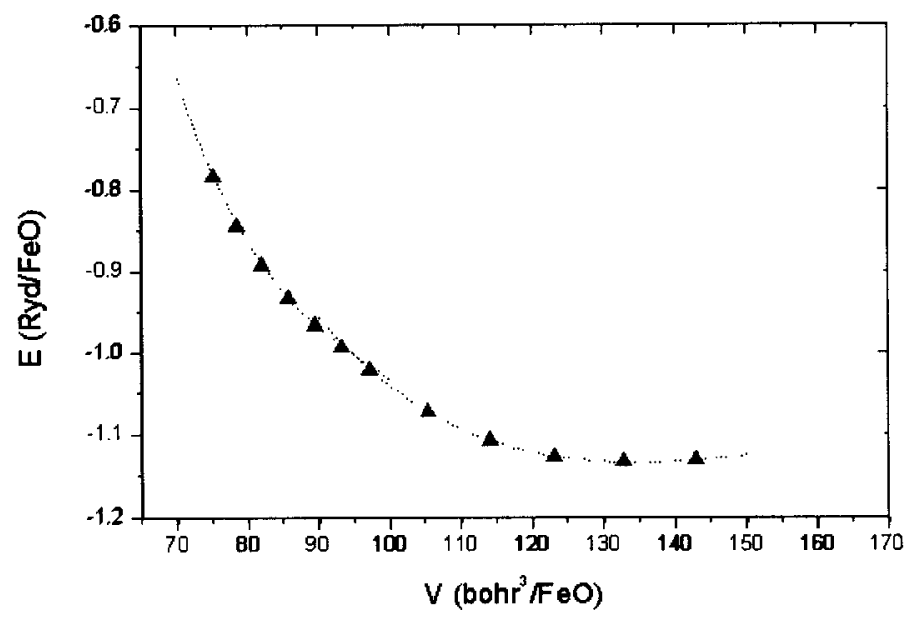

Figure 2. Energy versus volume for AFM FeO computed using LAPW and GGA PBE96. A dimple is evident in the total energies, indicating a first order phase transition. The dotted lines are Birch equation of state fits to the low pressure and high pressure data points. A first order transition occurs at the common tangent to these curves, as is indicated.

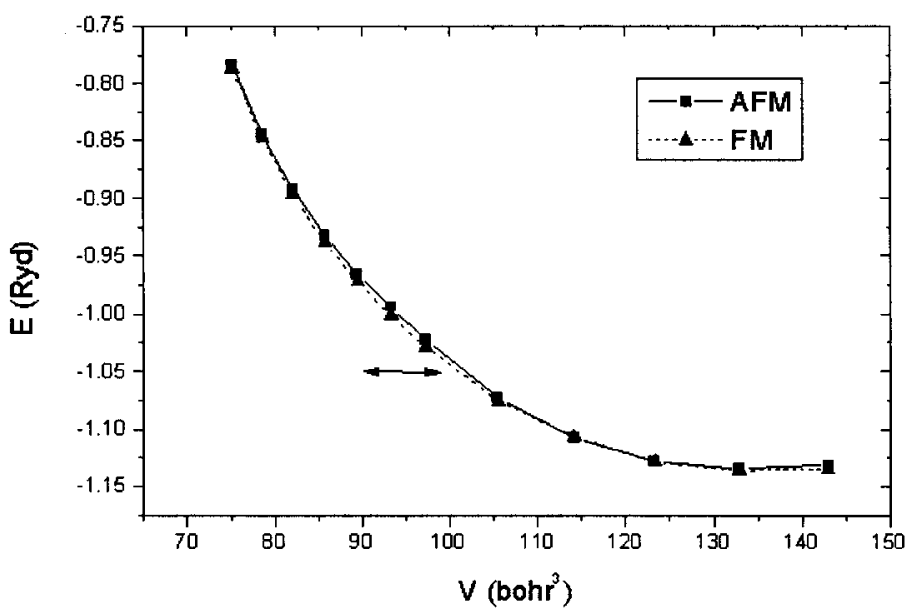

Figure 3. Energy versus volume for AFM and FM FeO computed using LAPW. FM is lower in energy in the region of the predicted first-order phase transition in AFM. The line with arrows indicates the volumes of the coexisting high-spin and low-spin AFM phases. 


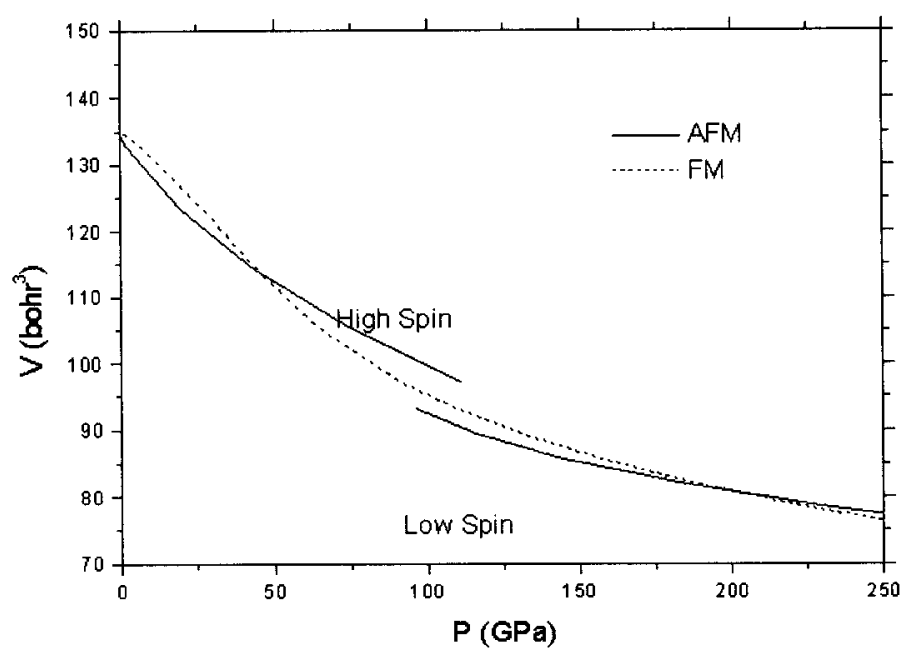

Figure 4. Volume versus pressure for AFM and FM FeO. Note that FM FeO is denser than high-spin AFM above $\sim 40 \mathrm{GPa}$, and is also lower in energy, so there may be a high pressure transition from AFM order to FM order at high pressures and low temperatures. The FM phase shows a continuous transition rather than a first-order phase transition. atom/cell ferromagnetic (FM) phase. Only cubic lattice parameters were studied here, as in the LMTO study. Our present results are significantly different from the LMTO results reported in Ref. [5]. We could not obtain two different AFM solutions at the same volume using the LAPW method as was found in LMTO. Figure 2 shows the energy versus volume computed with LAPW and GGA. The observed region of negative curvature in the energy volume curve is indicative of a first-order phase transition. The low pressure and high pressure regions can be fit separately to equations of state, as shown in fig. 2. The common tangent to the low pressure and high pressure regions indicates the first-order transition, and the slope of the tangent is $-\mathrm{P}$, which we find to be $105 \mathrm{GPa}$ with the LAPW. This contrasts with $200 \mathrm{GPa}$ found with LMTO, and is consistent with $\sim 100 \mathrm{GPa}$ found with LDA in the LAPW method in our earlier study [4]. The discrepancy we report here for the transition pressures in LAPW and LMTO is due to shifts in total energies, which are more sensitive to non-spherical changes in the charge density and potential which are neglected in LMTO-ASA. This seems to especially critical in the GGA, which is more sensitive to the non-spherical charge density, and the LMTO and LAPW LDA results are very close.

Interestingly, the FM solution is lower in energy than AFM in the range of the transition (fig. 3). The comparison of small differences in AFM and FM energies is extremely sensitive to
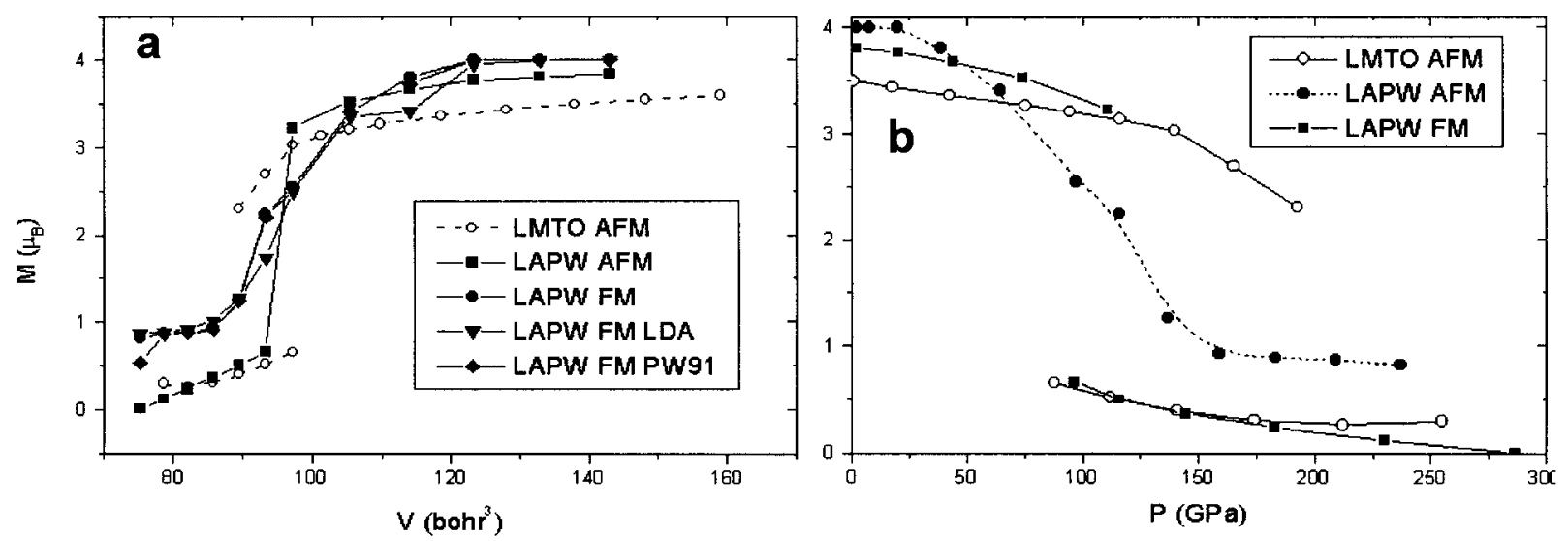

Figure 5. Magnetic moment per iron versus (a) volume and (b) pressure. The LAPW FM moments are the total moment per cell. The LAPW AFM moments are the moment in the muffin tin sphere renormalized by the ratio of the FM total moments to FM muffin tin moments, thus the LAPW AFM moments appear larger than the LMTO moments which are in the atomic sphere. The LAPW and LMTO moments agree quite well, showing that the difference between the transition in GGA between LAPW and LMTO is due to the approximations for the total energies in the LMTO-ASA. The high spin-low spin transition is discontinuous in AFM, and continuous in FM. Little difference is seen for different exchange correlation functionals. 
convergence, so the predicted stability of the FM ordering at high pressures should be regarded as preliminary. The results however suggest $T_{N}$ decreases with pressure after its initial rise [11]. Also, it will be important to consider the effects of strains on energetics and magnetic collapse; such computations are in progress.

Figure 4 shows volume versus pressure for AFM and FM FeO. There is a significant volume collapse at the phase transition in AFM FeO. However, the FM computations do not show a first order transition, but rather a continuous loss of moment. FM

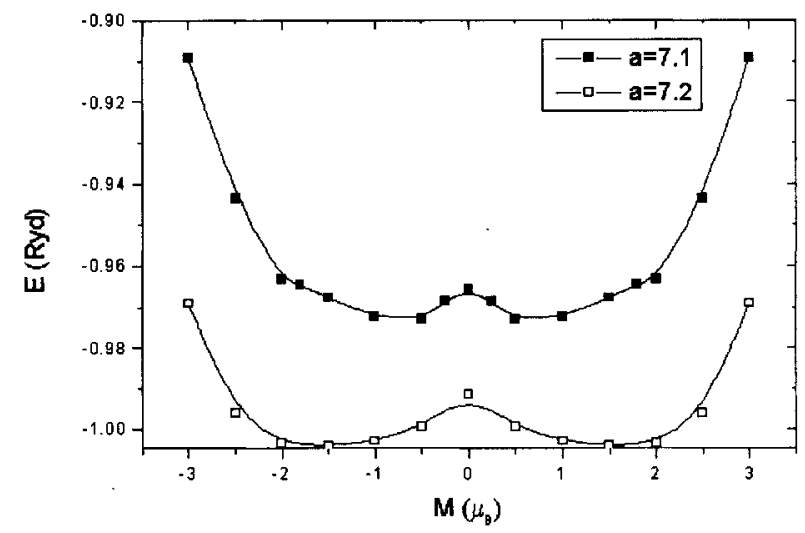

Figure 6. Results from fixed moment computations for $\mathrm{FM} \mathrm{FeO}$ at two volumes. The absence of multiple minima indicates a continuous transition. $\mathrm{FeO}$ is denser than $\mathrm{AFM} \mathrm{FeO}$ at moderate pressures (above $40 \mathrm{GPa}$ ) and is also lower in energy than AFM FeO, so there may be a magnetic phase transition from AFM to FM ordering at low temperatures and high pressures.

We obtained the AFM high-spin low-spin transition pressure by fitting the low-spin and high-spin results separately to second order Birch equations of state. This gives a transition

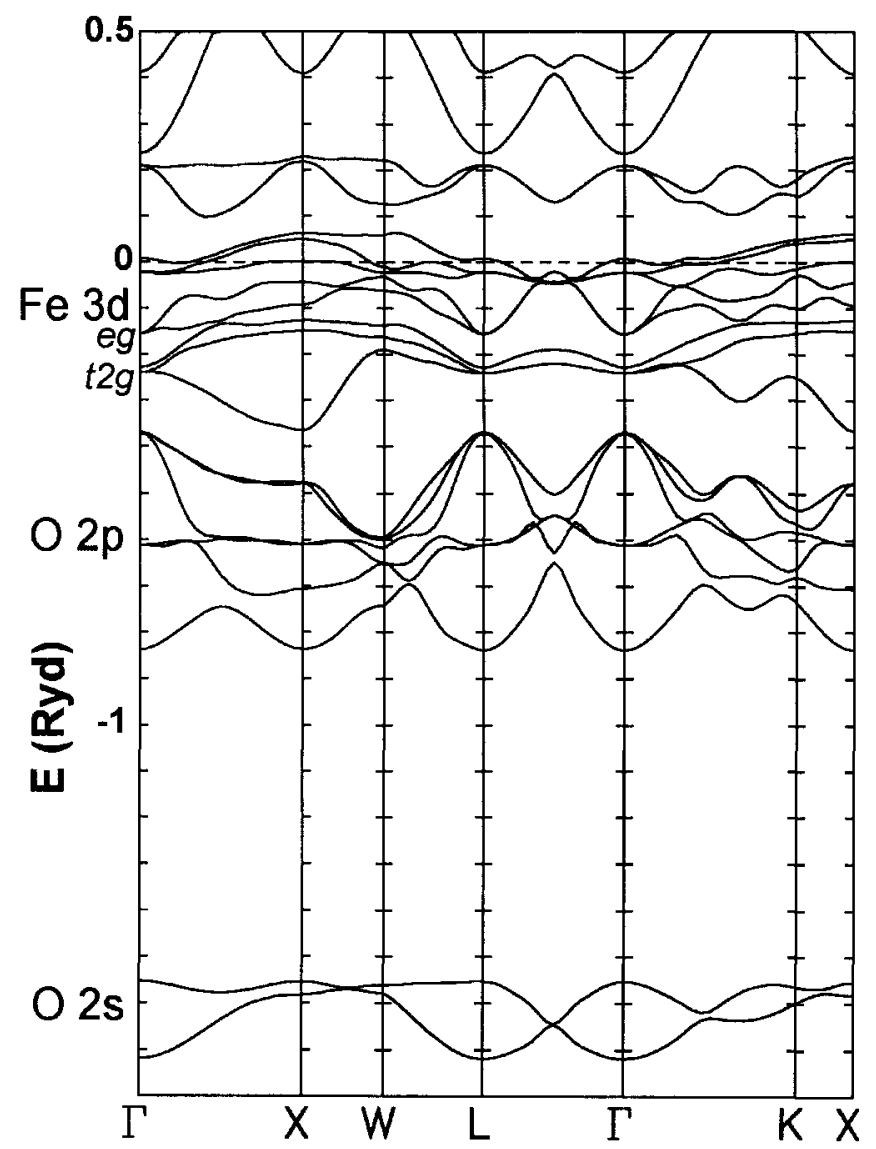

Figure 7. LAPW band structure of $\mathrm{AFM} \mathrm{FeO}$ for $\mathrm{a}=7.3 \mathrm{bohr}$, $87 \mathrm{GPa}$. pressure $105 \mathrm{GPa}$ with a $\Delta \mathrm{V}$ of $8 \%$ for AFM with a cubic lattice. This transition pressure may change when strains are included.

Figure 5 shows the moments versus volume and pressure. The LMTO results agree fairly well with LAPW in giving the moment versus volume, and we do not find the moments to be sensitive to the exchange correlation functional, whether LDA or GGA with PW91 or PBE96. As mentioned above, the FM moments are continuous as functions of pressure, and there are not simultaneous low-spin and highspin solutions. This is illustrated in fig. 6, which shows the results of fixed moment LAPW computations. There is only a single minimum (of given sign), whereas a discontinuous transition would lead to multiple minima.

Next we consider the origin of the high-spin low-spin transitions in these materials. Figure 7 shows the computed AFM band structure at a 
volume of 97.25 bohr $^{3}$ per $\mathrm{FeO}(\mathrm{a}=7.3 \mathrm{bohr})$. The notation for the cubic rocksalt structure is used rather than the magnetic symmetry to ease comparison with published band structures. The bands are folded (doubled) due to the doubling of the unit cell in the AFM phase. The lower $\mathrm{t}_{2 \mathrm{~g}}$ and $\mathrm{e}_{\mathrm{g}}$ states are for the majority spin at each $\mathrm{Fe}$ site at $\Gamma$. The small splitting of the $\mathrm{t}_{2 \mathrm{~g}}$ state is due to the magnetic structure. Above these are the minority spin $\mathrm{t}_{2 \mathrm{~g}}$ and $\mathrm{e}_{\mathrm{g}}$ states (unlabeled). Interestingly, the partially occupied upper set of $t_{2 \mathrm{~g}}$ triply degenerate at states at the Fermi level $E_{F}$, indicated by the dashed line, are much narrower than the lower set. These flat bands may be important in stabilizing the localized Mott state at low pressures.

The commonly accepted explanation is that crystal field splitting of $e_{g}$ and $t_{2 g}$ states increases with pressure due to the change in electrostatic potential from the ionic lattice [12-15]. This would give a $1 / r^{5}$ dependence of the splitting, which is close to what is observed in some materials [15]. We find, however, that the effects of the non-spherical electrostatic potential are extremely small, and are not responsible for the splitting of the $e_{g}$ and $t_{2 g}$ states. In fact, the LMTO-ASA does not even include this effect at all, and it shows almost identical splitting to the LAPW which does include all non-spherical contributions. Indeed, the pure electrostatic term even has the wrong sign. Instead, the observed $\mathrm{e}_{\mathrm{g}}$ and $\mathrm{t}_{\mathrm{gg}}$ splitting arises almost entirely from hybridization. In ref. [5], we argued that the high-spin low-spin transition is due to band widening with increasing pressure, rather than changes in electrostatic crystal field splitting, and interpreted the transition in terms of an extended Stoner model. The band widening arises from increased hybridization with pressure, and is a necessary result of the shortening of distances between atoms with increasing pressure. Pasternak et al. [6] said that they see no experimental evidence for band widening in high pressure Mössbauer measurements (see below), but this argument is unclear since they assume the moment and bandwidth must be proportional, which is not the case. We find the high-spin moment to vary little with pressure in AFM until the vicinity of the collapse, but the bands widen due to the ubiquitous increase in hopping integrals with decreasing bond distances.

The origin of crystal field splitting in hybridization, and not the crystal field, was discussed three decades ago in the seminal work on Mattheiss [16]. The $e_{g}-t_{2 g}$ splitting is due to offdiagonal interaction, and not shifts in the diagonal elements of the Hamiltonian matrix. The $\mathrm{e}_{\mathrm{g}}-$ $t_{2 g}$ splitting can be considered a ligand field effect, but is not at all related to changes in electrostatic field at the atomic site. Rather the splitting is due to $d$-d interactions between next nearest neighbor cations (at $\Gamma$ ), and due to $\mathrm{p}-\mathrm{d}$ and $\mathrm{s}-\mathrm{d}$ interactions with neighboring oxygen ions (throughout the Brillouin zone). The d-d interactions lead to splitting approximately as $1 / r^{5}$, and the $p-d$ and s-d interactions give splittings that would vary approximately as $1 / r^{7}$ [17]. The metal-metal $\mathrm{d}-\mathrm{d}$ splitting dominates. The observed $1 / \mathrm{r}^{5}$ dependence [15] of the so-called crystal field splitting does not prove the electrostatic crystal field model for the splitting, since the same dependence is given by hybridization effects.

We have computed the LAPW band structure with and without the electrostatic splitting by turning off all but the $l=0$ part of the potential inside the spheres (but using the full selfconsistent charge density). The band structure cannot be distinguished from the full potential band structure on the scale of fig. 7. The $t_{2 \mathrm{~g}}$ state does not move at all at $\Gamma$ (at -.37 Ryd from $\mathrm{E}_{\mathrm{F}}$, $1 \mathrm{Ryd}=13.605 \mathrm{eV}$ ), and the $\mathrm{e}_{\mathrm{g}}$ state (at $-.24 \mathrm{Ryd}$ from $\mathrm{E}_{\mathrm{F}}$ ) moves by 4 mRyd. The $\mathrm{t}_{2 \mathrm{~g}}-\mathrm{e}_{\mathrm{g}}$ splitting at $\Gamma$ is due to $d-d$ interactions between Fe ions, and the full band width is due also to interactions with $\mathrm{O} 2 p$. The small difference when omitting $l>0$ indicates that the splitting and band width is not due to electrostatic crystal field effects, but rather to hybridization, just as was described by Mattheiss [18]. Regarding the electrostatic crystal field effect., or so-called "point- 
charge model," Mattheiss stated "although the point-ion model often yields results which are in fair agreement with experiment, we tend to regard this success as accidental rather than fundamental [19]." This remains the case.

Recently Mössbauer measurements were reported that were interpreted as showing continuous magnetic collapse in FeO at pressures above $90 \mathrm{GPa}$ [6], very different from the LMTO predictions [5], but consistent with the present results and those of Isaak et al. Similar theoretical calculations for $\mathrm{NiI}_{2}$ [20] to ours are also in very good agreement with experiment, and show a high-spin low-spin transition similar to what we find here for the transition metal oxides. Interestingly, the difference between GGA and LDA in the $\mathrm{NiI}_{2}$ system which also used LAPW, was small, consistent with our present results.

Pasternak et al. [6] claimed that the previously observed transition in $\mathrm{NiI}_{2}$ is not a high-spin low-spin transition, but is a Mott or charge transfer transition. However, it seems most likely that both the $\mathrm{NiI}_{2}$ and $\mathrm{FeO}$ transitions are of the high-spin low-spin type. Pasternak et al. infer that there is no volume effect for the magnetic collapse, whereas we find an appreciable volume effect at the first-order transition. However, since high-spin and low-spin FeO can coexist if the transition is first-order, and a pressure gradient is present in the experiment, the transition may be apparently smeared out and the volume discontinuity difficult to observe. Alternatively, since we find that FM FeO will have a continuous transition, and find FM FeO lower in energy than AFM (at least for the cubic lattice), it is possible that FeO is FM at the experimental conditions, and the transition is indeed continuous. A third possible explanation is the fact that FeO is non-stoichiometric, and this may also smear out the transition.

A second interesting point is that Pasternak et al. find a low-spin (or non-magnetic) state at high temperatures, and a high-spin (or magnetic ordered) state at low temperatures. However, one might think the high-spin state should be the high entropy state, with a magnetic entropy of $\mathrm{R} \ln (M+1)$, and thus should be the high temperature state, all else being equal. On the other hand, FeO may have already undergone a insulator to metal transition by this pressure, and changes in the Fermi entropy with magnetic collapse may compensate for the pure magnetic entropy term. The key to understanding this may be the present results that show FM ordering stabilized over AFM with increasing pressure. Thus the Néel temperature may decrease with pressure after an initial increase, and the magnetic collapse transition at high pressures and $T_{N}$ at low pressures are actually two parts of the same transition line. This is exactly what is seen in Monte Carlo simulations of the Hubbard model [21]. How the magnetic transitions interact with structural distortions and transitions requires further theoretical and experimental study.

\section{HIGH PRESSURE B8 PHASE}

Experiments have shown that at about $70 \mathrm{GPa}$ and $1000 \mathrm{~K}$ there is a triple point between the B1 (rocksalt) structure, the rhombohedral phase which is distorted B1, and the B8 (NiAs) structure (fig. 1) [22]. Although the diffraction positions for latter phase agreed well with NiAs, the intensities did not agree well with the normal $\mathrm{NiAs}$ structure with $\mathrm{Fe}$ at the $\mathrm{Ni}$ site and $\mathrm{O}$ at the As site. Therefore, we have reanalyzed the experimental data and performed first-principles computations to better understand the high pressure phase [23]. The high pressure phase is hexagonal, with space group $\mathrm{P}_{3} / \mathrm{mmc}$, and cell parameters of $\mathrm{a}=2.574(2) \AA$ and $\mathrm{c}=5.172(4) \AA$ with one atom at $\left[\begin{array}{lll}0 & 0 & 0\end{array}\right]$ and the other at $\left[\begin{array}{llll}2 / 3 & 1 / 3 & 14\end{array}\right]$. It can be considered as a distorted hexagonal closest packed analogue of rocksalt. In NiAs structure, $\mathrm{Ni}$ is assigned to the position at the origin (with trigonal prism coordination), resulting in nearest neighbor $\mathrm{Ni}-\mathrm{Ni}$ separations 


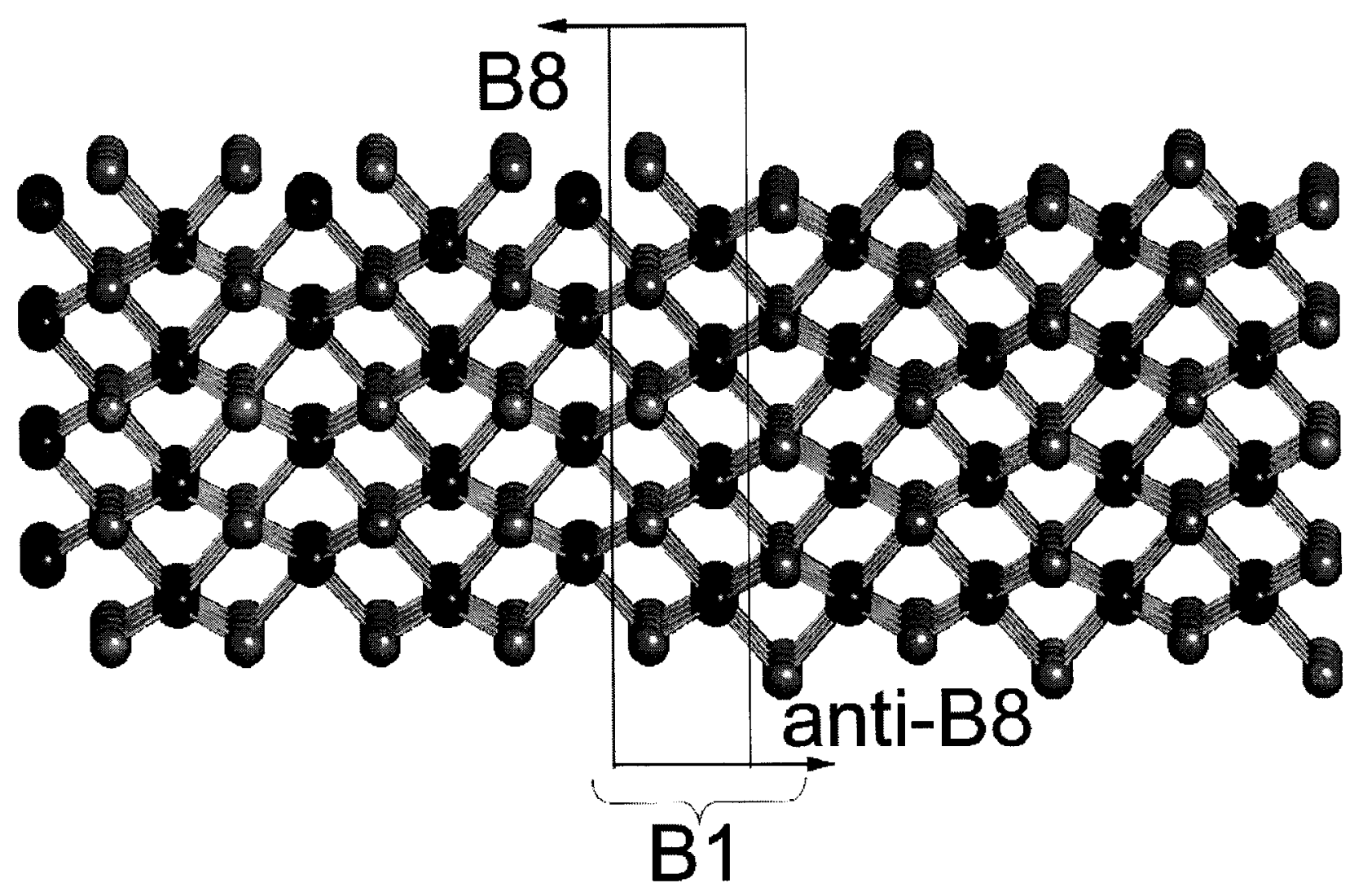

Figure 8. The 5:5 sequence of B8:anti-B8 polytype of FeO.

$(2.53 \AA)$ that are quite a bit smaller than those observed for As-As $(3.28 \AA)$. However, for $\mathrm{FeO}$ in the $\mathrm{B} 8$ structure, with a much larger $c / a$ ratio than $\mathrm{NiAs}$, the nearest neighbor $\mathrm{Fe}-\mathrm{Fe}$ distances and $\mathrm{O}-\mathrm{O}$ distances are the same at $2.57 \AA$. This removes the necessity of placing $\mathrm{Fe}$ in the trigonal prism sites, and so we considered both possibilities, the B8 structure, with $\mathrm{Fe}$ with trigonal prism coordination, and the anti-B8 structure, with Fe in octahedral coordination. Stacking faults are possible in a hexagonal close packed (hcp) structure, so we also considered polytypes. A variety of theoretical diffraction patterns were fit to the observed one with the final conclusion that the experimental diffraction pattern represented a 5:5 layered sequence of B8 and anti-B8 structures. Such a polytype as this appears to be unique in the sense that anions and cations are found substituting for each other. It was also possible to fit the diffraction pattern with a mechanical mixture of $\mathrm{B} 8$ and anti-B8, but this required the peaks widths of the anti-B8 to be over three-times larger than B8, and details of the spectra were not fit well. The stacking fault boundary between B8 and anti-B8 is the rhombohedrally distorted B1 structure (fig. 8), so we find that there can be continuous transitions among B1, B8, and anti-B8 by simply changing the amount of each stacking sequence.

We performed first-principles total energy calculations using the Linearized Augmented Plane Wave (LAPW) method under the Generalized Gradient Approximation (GGA) [10] (fig. 9). We found that the total energies of ferromagnetic B8 and anti-B8 are close in energy, consistent with the proposed polytype. However, antiferromagnetic anti-B8 was much lower in energy than B8, so much so that it is predicted to be the ground state structure. This is possibly another failure of band theory for these materials, due to the fact that B8 is predicted to be a 
metal and anti-B8 predicted to be an insulator by theory (see below). Two other possibilities must be considered. At low pressures, $\mathrm{B} 1 \mathrm{FeO}$ is nonstoichiometric, but the theory is performed for perfect stoichiometric $\mathrm{FeO}$. We found that the entropic stabilization of $\mathrm{Fe}_{1-\mathrm{x}} \mathrm{O}$ is unlikely to be responsible for the observed stability of $\mathrm{B} 1$ at low pressures, although it does contribute to its stability. Another important point though is that, although the high pressure Néel point $T_{N}$, where the antiferromagnetic order sets in, is unknown, it is quite probable that the experiments are performed above $\mathrm{T}_{\mathrm{N}}$ and the FM energetics are more appropriate. This is based on the discussion above that $T_{N}$ may break off from $T_{R}$, the rhom-

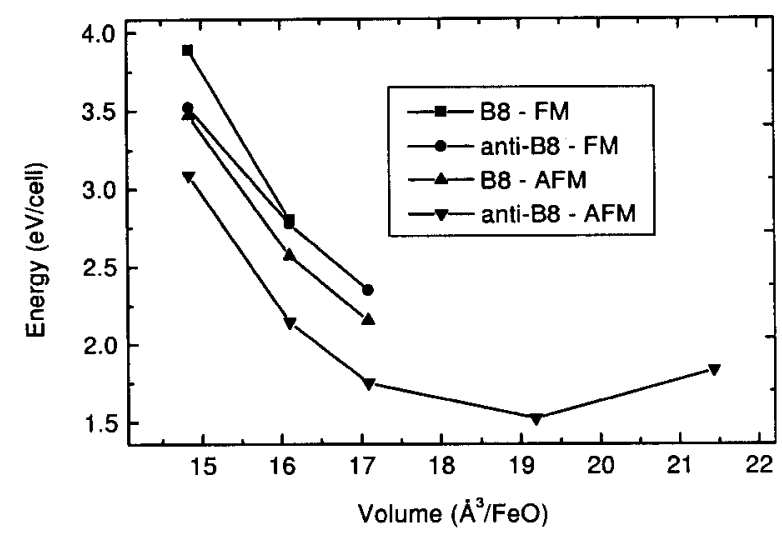

Figure 9. Total energies for ferromagnetic (FM) and antiferromagnetic (AFM) B8, anti-B8 structures computed using the LAPW method and GGA (PW-91). bohedral transition line, with increasing pressure, and turn around and then decrease with increasing pressure. This would be consistent with our finding that FM FeO is lower in energy than AFM at high pressures, though computations on strained FM and AFM FeO must be completed to verify this. Even at low pressures, below $T_{N}$ $(200 \mathrm{~K})[24]$, it is possible that anti-B8 becomes stable relative to B1, but there is insufficient kinetic energy for a reconstructive phase transition to occur. In any case, the phase diagram and properties of $\mathrm{FeO}$ are likely to be much richer as functions of $\mathrm{P}$ and $\mathrm{T}$ than previously assumed.

The band structure of anti-B8 is insulating, alone among FeO structures that have been studied theoretically. $\mathrm{FeO}$ is a Mott insulator, and conventional band theory predicts $\mathrm{FeO}$ to be a metal at low pressures. At high pressures $\mathrm{FeO}$ would be a band metal after undergoing a Mott transition. Band widening would lead to this transition, probably before or at the magnetic collapse pressure [5]. We find B8 and anti-B8 to be antiferromagnetic, but B8 is metallic and anti-B8 is insulating [23]. Experiments have shown that the high pressure hexagonal phase is metallic [25]. A stacking polytype of B8 and anti-B8 would have metallic and insualting layers, leading to very anisotropic conductivity.

\section{GEOPHYSICAL IMPLICATIONS}

The behavior of transition metal oxides and other transition metal ion bearing materials have important implications for the Earth. The collapse of the spin moments for some metal ions at elevated pressure has implications on elastic properties and equations of state in Earth's lower mantle, and especially the core and $\mathrm{D}^{\prime \prime}$ region (the region of the mantle just above the core) which is probably enriched in $\mathrm{FeO}$, and complicate attempts to understand chemical bonding in the deep Earth. These complications ultimately must be addressed to unravel questions about Earth's composition and differentiation, the structure of the region around the coremantle-boundary (CMB), thermal diffusivity, and electrical conductivity.

Our results may be relevant to the structure and dynamics of the $\mathrm{D}^{\prime \prime}$ side of the CMB. Seismologically determined inhomogeneities in the lowermost mantle are usually explained in terms of partial melting, chemical inhomogeneities, and/or solid-solid mineral phase transitions $[26,26]$. While some recent studies find velocity changes in $\mathrm{D}^{\prime \prime}$ consistent with zones of partial melting [27,28], Franck and Kowalle find evidence for chemical inhomogeneities or some 
combination of chemical and thermal effects [29]. Experiments at high pressure and temperature have demonstrated that reaction products of metallic alloy and non-metallic silicates result from mixing of liquid iron and silicates and oxides [30-32]. The essential point is that these experiments show that oxides and molten iron react at high pressure and temperature, suggesting $\mathrm{FeO}$ is extracted from the oxide, thus providing a mechanism for light elements (oxygen) in the core and explaining how a region $\left(\mathrm{D}^{\prime \prime}\right)$ with chemical heterogeneity can occur. Thus $\mathrm{D}^{\prime \prime}$ is probably enriched in a $\mathrm{FeO}$ phase, which is probably the anti-B8 or B8-anti-B8 polytypes as we have discussed above. Such phases would have high metallic conductivity that is highly anisotropic, and would be important for determining how the magnetic field is propagated up from the core.

Another area that our results will impact in the long standing problem of the excess siderophile abundances in Earth's mantle relative to the lithophile elements. $\mathrm{Co}, \mathrm{Fe}, \mathrm{Mn}$, and $\mathrm{Ni}$ are four such siderophile elements. A resolution of this problem was provided in part by considering the temperature effect on the partition coefficients[33], but temperature alone may not provide a complete resolution of this problem [34]. Our results indicate, however, that changes in the nature of bonding brought on by magnetic collapse at high pressure also can affect the partition coefficients $\left(\mathrm{K}_{\mathrm{d}}\right)$ of some siderophile elements. In this scenario extrapolation of low pressure results may not be accurate for some siderophiles at elevated pressure.

Thus we see that electronic changes such as magnetic collapse could have important implications for the geochemistry and geophysical properties of the deep Earth. Structural transitions such as the transition to anti-B8 or B8 in $\mathrm{D}^{\prime \prime}$ could also change phase relations and help contribute to the heterogeneity of this region[35,36]. Phase transitions to metallic phases would be very important in influencing the electrical and thermal boundary conditions of the Earth's mantle, and contribute to dynamic processes such as plumes that give rise to volcanic islands such as Hawaii[37].

\section{SUMMARY}

The behavior of transition metal oxides at high pressures is a key problem for a number of fields, including condensed matter physics and geophysics. The richness of behavior of these materials as functions of pressure and temperature is only beginning to be known. Study of these systems pushes both theory and experimental techniques to their current limits. Understanding of the behavior of the transition metal oxides is a challenge that will require, and lead to, significant advances in theory and experimentation.

\section{ACKNOWLEDGMENTS}

This work is supported by National Science Foundation Grant EAR-9418934 (REC), EAR9418945 (YF), and the NSF Center for High Pressure Research. Computations were performed on the Geophysical Laboratory Cray J916/16-4096 supported by NSF-EAR-9512627. Partial support (DI) was also provided by the Office of Naval Research. We thank V.I. Anisimov, R.M. Hazen, A.I. Liechtenstein, H.-K. Mao and C.T. Prewitt for helpful discussions. 


\section{REFERENCES}

1. N.F. Mott, Metal-Insulator Transitions, 286 pp., Taylor \& Francis, New York, 1990.

2. J. Ita and R.E. Cohen, Phys. Rev. Lett., 79, 3198 (1997).

3. M.J. Mehl, R.E. Cohen, and H. Krakauer, J. Geophys. Res., 93;94;1989, 8009;1977 (1988).

4. D.G. Isaak, R.E. Cohen, M.J. Mehl, and D.J. Singh, Phys. Rev. B, 47, 7720 (1993).

5. R.E. Cohen, I.I. Mazin, and D.G. Isaak, Science, 275, 654 (1997).

6. M.P. Pasternak, R.D. Taylor, R. Jeanloz, X. Li, J.H. Nguyen, and C. McCammon, Phys. Rev. Lett., 79, 5046 (1997).

7. S.H. Wei and H. Krakauer, Phys. Rev. Lett., 55, 1200 (1985).

8. J.P. Perdew and Y. Wang, Phys. Rev. B, 45, 13244 (1992).

9. O.K. Andersen, Phys. Rev. B, 12, 3060 (1975).

10. J.P. Perdew, K. Burke, and M. Ernzerhof, Phys. Rev. Lett., 77, 3865 (1996).

11. G. Zou, H.-K. Mao, P.M. Bell, and D. Virgo, Carnegie Institution of Washington Year Book, 79, 374 (1980).

12. R.G. Burns, Mineralogical Applications of Crystal Field Theory, 551 pp., Cambridge University Press, Cambridge, 1993.

13. H. Watanabe, Operator Methods in Ligand Field Theory, 193 pp., Prentice-Hall, Englewood Cliffs, New Jersey, 1966.

14. C.B. Bargeron, M. Avinor, and H.G. Drickamer, Inorganic Chemistry, 10, 1338 (1971).

15. H.G. Drickamer and C.W. Frank, Electronic Transition and the High Pressure Chemistry and Physics of Solids, 220 pp., Chapman and Hall, London, 1973.

16. L.F. Mattheiss, Phys. Rev. B, 5, 290 (1972).

17. W.A. Harrison, Electronic Structure and the Properties of Solids: The Physics of the Chemical Bond, 582 pp., W. H. Freeman and Company, San Francisco, 1980.

18. L.F. Mattheiss, Phys. Rev. B, 5, 306 (1972). 
19. L.F. Mattheiss, Phys. Rev. B, 2, 3918 (1970).

20. P. Dufek, P. Blaha, and K. Schwarz, Phys. Rev. B, 51, 4122 (1995).

21. R.T. Scalettar, D.J. Scalapino, R.L. Sugar, and D. Toussaint, Phys. Rev. B, 39, 4711, 1998.

22. Y. Fei and H.-K. Mao, Science, 266, 1668 (1994).

23. I.I. Mazin, Y. Fei, J.W. Downs, and R.E. Cohen, Amer. Mineral., in press, 1998.

24. C. McCammon, J. Magn. Magn. Mat., 104-107, 1937 (1992).

25. E. Knittle and R. Jeanloz, Geophys. Res. Lett., 13, 1541 (1986).

26. Q. Williams and E.J. Garnero, Science, 273, 1528 (1996).

27. J.M. Kendall and P.G. Silver, Nature, 381, 409 (1996).

28. J. Revenaugh and R. Meyer, Science, 277, 670 (1997).

29. S. Franck and G. Kowalle, Phys. Earth Planet. Inter., 90, 157 (1995).

30. E. Knittle and R. Jeanloz, Science, 251, 1438 (1991).

31. E. Knittle and R. Jeanloz, Geophys. Res. Lett., 16, 609 (1989).

32. F. Goarant, F. Guyot, J. Peyronneau, and J. Poirier, J. Geophys. Res., 97, 4477 (1992).

33. V. Ramamurthy, Science, 253, 303 (1991).

34. D. Walker, L. Norby, and J.H. Jones, Science, 262, 1858 (1993).

35. R. Jeanloz and T. Lay, Scientific American, 268, 26 (1993).

36. R. Jeanloz and B. Romanowicz, Physics Today, August, 22 (1997).

37. M. Manga and R. Jeanloz, Geophys.Res.Lett., 23, 3091 (1996). 